Short Communication

\title{
Large-scale Synthesis of $\mathrm{Cu}_{2} \mathrm{O}$ Nanocubes and Their Electrochemical Properties
}

\author{
Hongdong Liu ${ }^{l}$, Zhongli Hu ${ }^{l}$, Rong Hu ${ }^{l, *}$, Bitao Liu ${ }^{l}$, Haibo Ruan ${ }^{1, *}$, Lei Zhang ${ }^{2}$,Wei Xiao ${ }^{l}$ \\ ${ }^{1}$ Research institute for new materials technology, Chongqing university of arts and sciences, \\ Chongqing 402160, PR China \\ ${ }^{2}$ College of life science, Chongqing normal university, Chongqing 401331, PR China \\ *E-mail: hurong_82@163.com, rhbcqu@aliyun.com
}

doi: $10.20964 / 110402756$

Received: 5 January 2016 / Accepted: 26 January 2016 / Published: 1 March 2016

\begin{abstract}
As an environmentally-friendly $p$-type semiconductor, cuprous oxide $\left(\mathrm{Cu}_{2} \mathrm{O}\right)$ has exhibited potential applications, in particular sensors, solar cells, and $\mathrm{Li}$-ion batteries. In this paper, uniform $\mathrm{Cu}_{2} \mathrm{O}$ nanocubes were large-scale fabricated through a solution-based chemical precipitation method. The assynthesized products were characterized by XRD and SEM. As anode materials for Li-ion batteries, $\mathrm{Cu}_{2} \mathrm{O}$ nanocubes show excellent cycling performance, high capacity retention and coulombic efficiency.
\end{abstract}

Keywords: Cuprous oxide, Li-ion batteries, nanocubes, electrochemical performance

\section{$\underline{\text { FULL TEXT }}$}

(C) 2016 The Authors. Published by ESG (www.electrochemsci.org). This article is an open access article distributed under the terms and conditions of the Creative Commons Attribution license (http://creativecommons.org/licenses/by/4.0/). 